\title{
Expression of transient receptor potential melastatin 4 in differential diagnosis of eosinophilic renal tumors
}

\author{
GANIME ÇOBAN $^{1}$, PELIN YILDIZ $^{1}$, BAYRAM DOĞAN $^{2}$, NURHAN ŞAHIN $^{1}$ and ZÜHAL GÜCIN ${ }^{1}$ \\ Departments of ${ }^{1}$ Pathology and ${ }^{2}$ Urology, Faculty of Medicine, Bezmialem Vakif University, Istanbul 34093, Turkey
}

Received November 19, 2020; Accepted July 2, 2021

DOI: $10.3892 / \mathrm{mco} .2021 .2393$

\begin{abstract}
Immunohistochemical and molecular studies to differentiate eosinophilic kidney tumors are gradually increasing. The present study investigated the role of transient receptor potential cation channel subfamily $\mathrm{M}$ member 4 (TRPM4), a non-selective cation channel associated with migration, proliferation and invasion in cancer cells, in this differentiation. The aim was to investigate the effectiveness of TRPM4 in differentiation of eosinophilic kidney tumors. The study included a total of 112 patients, including 97 eosinophilic kidney tumors with the diagnoses of 33 eosinophilic clear cell renal cell carcinoma (CCRCC), 35 eosinophilic chromophobe renal cell carcinoma (ChRCC), 8 papillary renal cell carcinoma type 2 (P2RCC), 21 renal oncocytoma (RO), as well as 15 papillary renal cell carcinoma type 1 to differentiate from P2RCC. For TRPM4, diffuse staining ( $>10 \%)$ was observed in 2 CCRCC, 15 ChRCC, 20 RO and 4 P2RCC cases. There was a significant difference between eosinophilic CCRCC and other eosinophilic tumors $(\mathrm{P}<0.05)$. While basolateral staining was observed in papillary tumors, membrane staining was observed in other stained cases. It was hypothesized that the use of TRPM4 along with morphological findings, cytokeratin 7 and other markers may be useful for the differentiation of eosinophilic kidney tumors.
\end{abstract}

\section{Introduction}

Renal cell carcinoma accounts for approximately $2-3 \%$ of adult cancers, and the rate of its incidental diagnosis is increasing with the increased use of imaging methods. Numerous diagnostic, predictive and prognostic biomarkers have been studied for the diagnosis, treatment planning, and follow-up. Especially the differentiation of eosinophilic kidney tumors has become more challenging with newly defined tumors and the need for

Correspondence to: Dr Ganime Çoban, Department of Pathology, Faculty of Medicine, Bezmialem Vakif University, Adnan Menderes Boulevard, Fatih, Istanbul 34093, Turkey

E-mail: drgcoban@hotmail.com

Key words: eosinophilic renal cell carcinoma, transient receptor potential melastatin 4 , immunohistochemistry, oncocytoma different immunohistochemical and molecular-genetic studies for differential diagnosis has increased day by day $(1,2)$. The marker thought to help in the differentiation of these tumors is TRPM4 (transient receptor potential cation channel subfamily M4), a protein localized on the cell membrane.

In mammals, there are 28 TRP (transient receptor potential) channels, which are cation-selective and located on the cell membrane. Although this protein superfamily has 6 subgroups, one of the most important is TRP that contains Melastatin (3). TRPM (transient receptor potential cation channel subfamily $\mathrm{M}$ ) has eight subgroups, most of which are permeable to bivalent cations, while TRPM4 and TRPM5 are channels that are impermeable to calcium $\left(\mathrm{Ca}^{+2}\right)$ but only permeable to monovalent cations. When the amount of cytosolic ATP decreases and $\mathrm{Ca}^{+2}$ level increases, the TRPM4 channel is activated (4) and indirectly help regulate intracellular $\mathrm{Ca}^{+2}$ through the regulation of cell membrane potential. These ion channels and $\mathrm{Ca}^{+2}$ are responsible for cell proliferation, apoptosis, and differentiation to maintain cell homeostasis (5). The expression of TRPM4 has been evaluated in cancer of several organs, especially the prostate and colon $(6,7)$. However, there is no clear data regarding the place of the kidney in the differential diagnosis of eosinophilic tumors.

The aim of our study was to analyze the staining pattern of TRPM4 antibody in common eosinophilic kidney tumors such as eosinophilic clear cell renal cell carcinoma (CCRCC), Chromophobe Renal Cell Carcinoma (ChRCC), Renal Oncocytoma (RO) and Papillary Renal Cell Carcinoma Type 2 (PRCC2), and to determine whether it can be a diagnostic marker.

\section{Materials and methods}

Patient. The study included a total of 112 patients, including 97 patients diagnosed with eosinophilic CCRCC, ChRCC, P2RCC, RO and 15 patients with P1RCC from the partial and radical nephrectomy specimens studied at Bezmialem Vakif University Faculty of Medicine between January 2014 and 2019. This study is a retrospective study made of paraffin blocks. The presence of TRPM4 could also be demonstrated by Western Blot analysis; however, Western Blot analysis could not be performed in this study as there were no fresh tissues of the cases. The study was approved by the ethics committee board of Bezmialem University. 
Immunohistochemistry. In the present study, hematoxylin and eosin (H\&E) stained preparations were re-evaluated, and block selection was made for immunohistochemical staining. Two-micron thick slides were taken from the paraffin blocks prepared from formalin-fixed specimens obtained from the primary tumor. On these slides, TRPM4 (monoclonal mouse antibody; Novus Biologicals, USA Clone OTI10H5) immunohistochemistry was performed by being diluted at a ratio of $1 / 100$ in $2 \mathrm{~h}$ of incubation in Ventana Benchmark Ultra and Bench Mark XT devices.

Scoring of immunoreactivity. The extent of TRPM4 staining was evaluated semi-quantitatively and interpreted as 0 , negative; 1 , focal positive (1-10\%); and 2, diffuse positive staining (more than 10\%). Staining patterns were evaluated as membranous-cytoplasmic and basolateral. Staining patterns and their extent were compared with tumor types, demographic data, and cytokeratin 7 (CK7) staining status.

Statistical analysis. The statistical analysis was evaluated using the IBM SPSS 22.0 statistical software package. The distribution of categorical variables was evaluated with the Chi-square test. Descriptive statistics were expressed as standard deviation, frequency, and percentage. A P-value of $<0.05$ was considered to indicate a statistically significant difference.

\section{Results}

Patient data and histopathological parameters. Of the 112 patients included in the study, 33 (29.5\%) had eosinophilic CCRCC, 35 (31.3\%) had eosinophilic ChRCC, 8 (7.1\%) had P2RCC, 21 (18.8\%) had RO, and 15 (13.4\%) had P1RCC. Of the patients, $46(41.1 \%)$ were female and $66(58.9 \%)$ were male, with a mean age of 57.8 years. The clinical and pathological parameters of the patients are summarized in Table I.

TRPM4 staining rates and distributions in CCRCC, ChRCC, $R O$ and $P 2 R C C$ tumors. The staining ratios of the tumors with TRPM4 are presented in Table II. Among the 97 eosinophilic tumors, $2(4.9 \%)$ of the 41 patients with diffuse staining for TRPM4 were CCRCC, 15 (36.6\%) were ChRCC, 20 (48.8\%) were RO, and 4 (9.8\%) were P2RCC. While a significant result was obtained between the RO and ChRCC and CCRCC and $\mathrm{P} 2 \mathrm{RCC}$ patients $(\mathrm{P}=0.043)$, there was no significant result between the RO and ChRCC patients $(\mathrm{P}=0.065)$.

While TRPM4 staining was diffuse membranous in the collecting ducts of the healthy kidney, different staining patterns were observed in the oncocytoma and eosinophilic RCC subtypes. Staining was observed in 57 of the patients. Membranous staining was observed in 50 patients and basolateral staining in 7 patients. Of the patients with membranous staining, 26 (52\%) had ChRCC, 21 (42\%) had RO, 3 (6\%) had P2RCC. Membranous staining was not observed in any of the CCRCC patients, and there was a statistically significant difference between the RO and ChRCC patients $(\mathrm{P}<0.005)$. There were 2 (28.6\%) CCRCC, 1 (14.3\%) ChRCC, and 4 (57.1\%) P2RCC patients with basolateral staining. In terms of P1RCC, $1(6.7 \%)$ patient had negative staining, $3(20 \%)$ patients had focal, and $11(73.3 \%)$ patients had diffuse staining. Of the
P1RCC patients, 11 (73.3\%) had basolateral and 3 (20\%) had membranous staining (Fig. 1).

TRPM4 staining (negative, focal, diffuse staining) of the patients were compared with CK7 immunohistochemistry, which is used for the diagnosis and differentiation of tumors. CK7 and TRPM4 staining by the diagnoses of the tumors are summarized in Fig. 2. In CCRCC, focal staining was observed in $5(15 \%)$ cases with CK7 and diffuse staining in $2(4.9 \%)$ cases with TRPM4. In RO, focal staining was observed in $13(61.9 \%)$ cases with CK7, diffuse staining in 1 (4.7\%) case, diffuse staining in $20(86.9 \%)$ cases with TRPM4, and focal staining in $1(8.6 \%)$ case. ChRCC had diffuse staining in $34(97.1 \%)$ cases with CK7, focal staining in 1 (2.8\%) case, diffuse staining in $15(42.8 \%)$ cases with TRPM4, and focal staining in $12(34.2 \%)$ cases. In P2RCC, diffuse staining was observed in $3(37.5 \%)$ cases with CK7 and focal staining in 3 $(37.5 \%)$ cases, while diffuse staining was observed in $4(50 \%)$ cases with TRPM4 and focal staining in 3 (37.5\%) cases.

\section{Discussion}

$\mathrm{Ca}^{+2}$ dependent signaling pathways are associated with proliferation, migration, invasion, metastasis, and apoptosis of tumor cells $(8,9)$. TRPM4 is a monovalent nonselective cation channel activated by decreased ATP level and increased $\mathrm{Ca}^{+2}$ in case of hypoxia, the membrane is depolarized, and the voltage-dependent calcium channel is blocked by the $\mathrm{Na}^{+}$ current, decreasing the permeation of $\mathrm{Ca}^{+2}(10)$.

The expression of TRPM4 has been studied in multiple sclerosis (11), subarachnoid haemorrhage (12), and cerebral infarction (13), but there are very few studies on the relationship between the tumor and TRPM4 (14). It has recently been evaluated in prostate (15), bladder (16), colorectal cancer (17), cervical cancer (18), large B-cell lymphoma (19), and liver cancer (20). It has been determined that it is higher in the tumor in the prostate compared to healthy tissues, and its expression increases $(21,15)$ in the transition from prostatic intraepithelial neoplasia to prostatic carcinoma, and high levels of TRPM4 are associated with recurrence after prostatectomy (22). It has been found to be highly expressed in cervical cancer and large B-cell lymphoma compared to reactive tissues $(18,19)$. No difference has been found in the expression of TRPM4 between carcinoma of the bladder and healthy tissue (16). In our study, diffuse membranous staining was detected in healthy tissue and oncocytoma, while loss of expression was observed in CCRCC and different rates of staining were observed in ChRCC and P2RCC. In addition to healthy tissue, its staining in $\mathrm{RO}$ and ChRCCs suggests that it may be related to the same origin.

Over the past two decades, there have been many morphological, immunohistochemical and prognostic innovations in renal tumors (23). Among tumors with eosinophilic cytoplasm, there are new entities such as SDH-deficient RC, eosinophilic solid and cystic RCC, Warthin-like papillary RCC, and low grade oncocytic tumor in addition to ChRCC, RO, P2RCC, eosinophilic CCRCC $(2,23)$. There are a limited number of cases regarding these tumors, and studies are ongoing in terms of both diagnosis and prognosis. It is important to differentiate the oncocytoma, which accounts for approximately $10 \%$ of renal tumors, from malignant tumors, especially in needle 
Table I. Clinicopathological data of patients.

\begin{tabular}{|c|c|c|c|c|}
\hline Variable & $\operatorname{CCRCC}(n=33)$ & $\operatorname{ChRCC}(\mathrm{n}=35)$ & $\mathrm{RO}(\mathrm{n}=21)$ & $\mathrm{P} 2 \mathrm{RCC}(\mathrm{n}=8)$ \\
\hline Age, years $($ mean $\pm S D)$ & $58.97 \pm 12.5$ & $56.43 \pm 11.6$ & $57.14 \pm 13.38$ & $59.13 \pm 12.69$ \\
\hline \multicolumn{5}{|l|}{ Sex, n (\%) } \\
\hline Male & $21(63.6)$ & $15(42.9)$ & $10(47.6)$ & $6(75.0)$ \\
\hline Female & $12(36.4)$ & $20(57.1)$ & $11(52.4)$ & $2(25.0)$ \\
\hline Tumor size, cm (mean \pm SD) & $6.03 \pm 2.98$ & $5.85 \pm 0.85$ & $4.51 \pm 2.98$ & $6.81 \pm 2.25$ \\
\hline \multicolumn{5}{|l|}{ Tumour location, n (\%) } \\
\hline Left & $12(36.4)$ & $19(54.3)$ & $11(52.4)$ & $3(37.5)$ \\
\hline Right & $21(63.6)$ & $16(45.7)$ & $10(47.6)$ & $5(62.5)$ \\
\hline \multicolumn{5}{|l|}{$\mathrm{pT}, \mathrm{n}(\%)$} \\
\hline pT1 & $16(48.5)$ & $15(42.9)$ & & $3(37.5)$ \\
\hline pT2 & $5(15.2)$ & $13(37.1)$ & & $2(25.0)$ \\
\hline pT3 & $11(33.3)$ & $6(17.1)$ & & $2(2.5)$ \\
\hline pT4 & $1(3.0)$ & $1(2.9)$ & & $1(12.5)$ \\
\hline
\end{tabular}

CCRCC, clear cell renal cell carcinoma; RO, renal oncocytoma; ChRCC, chromophobe renal cell carcinoma; P2RCC, papillary renal cell carcinoma type 2 .

Table II. Transient Receptor Potential Melastatin 4 staining rates in eosinophilic kidney tumors.

\begin{tabular}{lcrr}
\hline Tumor numbers & Negative, $n(\%)$ & Focal positive, $n(\%)$ & Diffuse positive, $\mathrm{n}(\%)$ \\
\hline CCRCC $(\mathrm{n}=33)$ & $31(93.9)$ & $0(0.0)$ & $2(6.1)$ \\
ChRCC $(\mathrm{n}=35)$ & $8(22.9)$ & $12(34.3)$ & $15(42.9)$ \\
RO $(\mathrm{n}=21)$ & $0(0.0)$ & $1(4.8)$ & $20(95.2)$ \\
P2RCC $(\mathrm{n}=8)$ & $1(4.9)$ & $3(37.5)$ & $4(50.0)$ \\
\hline
\end{tabular}

CCRCC, clear cell renal cell carcinoma; RO, renal oncocytoma; ChRCC, chromophobe renal cell carcinoma; P2RCC, papillary renal cell carcinoma type 2 .

biopsies. In addition to morphological findings, CK7 is the most important marker in differentiating $\mathrm{ChRCC}$, another CD117-positive eosinophilic tumor $(2,24)$. While the staining pattern of $\mathrm{CK} 7$ is negative or focal positive in $\mathrm{RO}$, it is usually diffuse positive in ChRCC. In our study, the staining of TRPM4 expression was diffuse positive in oncocytoma, unlike CK7. But negative, focal positive and diffuse positive stainings were detected in ChRCC. Besides morphological findings in the differentiation between eosinophilic CCRCC and ChRCC, the use of stains such as CK7, CD117 and Carbonic Anhydrase IV along with TRPM4 will be supportive in the differentiation.

Papillary RCC has an incidence rate of $15 \%$ and consists of type 1 with amphophilic cytoplasm and type 2 with eosinophilic cytoplasm (25). TRPM4 generally showed diffuse basolateral staining in P2RCC, and the staining pattern was completely different from other eosinophilic tumors included in the study. Among papillary RCCs, P2RCC has worse prognosis than P1RCC, and it is important to differentiate between them. Diffuse positive basolateral staining was usually seen for TRPM4 in both of them. Although TRPM4 is not a marker that can be used to differentiate between
P1RCC and P2RCC, it is interesting that basolateral staining is dominant in papillary structuring.

There are publications in the literature showing an increase in the expression of TRPM4 in prostate, cervix, and large cell lymphoma. In these studies, there are arguments that suppression of TRPM4 can prevent tumor growth and metastasis (26). Wong and Hussain (27) also found TRPM4 to be associated with poor prognostic parameters in breast carcinoma. However, in this study, different expressions of TRPM4 were observed in malignant tumors of the kidney, while diffuse strong expression was remarkable in healthy tissue and oncocytoma, a benign tumor. In this study, TRPM4 was studied only for diagnostic purposes, although no interpretation could be made regarding the treatment or prognosis in $\mathrm{RCCs}$, it is thought that TRPM4 can be used together with other immunohistochemical markers in eosinophilic renal tumors.

In this study, it was concluded that TRPM4 was useful in the differentiation of eosinophilic kidney tumors. The extent of staining along with CK7 may be helpful in the differentiation between common oncocytoma and chromophobe RCC, and for the diagnosis of papillary RCC, it can be helpful in cases of difficulty with different staining patterns. In this respect, 
A

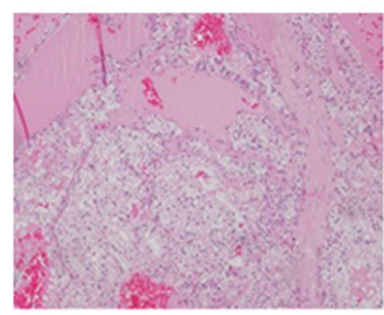

E

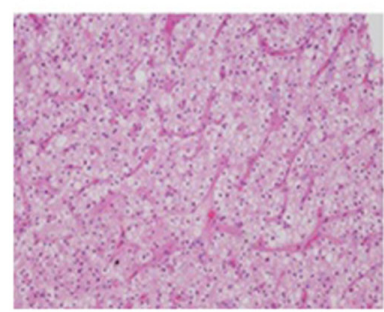

B

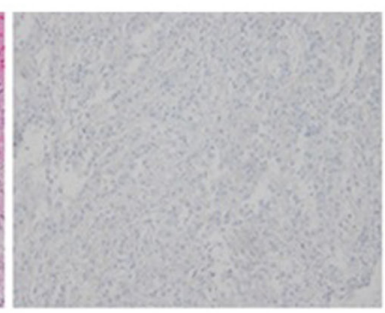

F

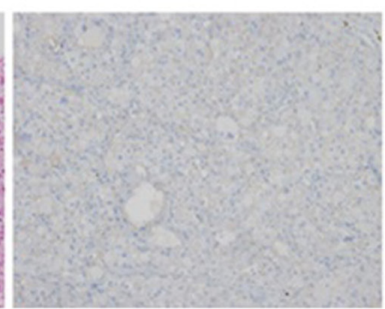

C

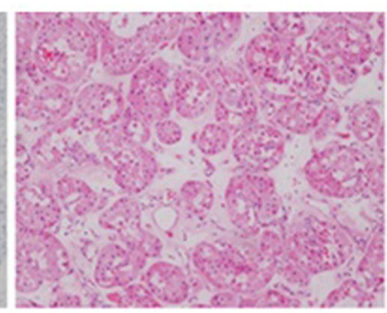

G

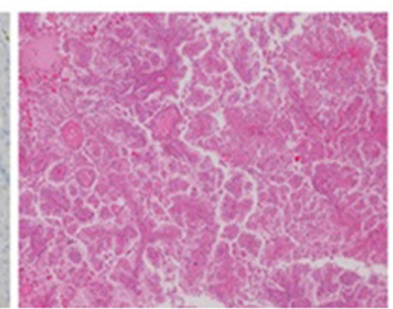

D

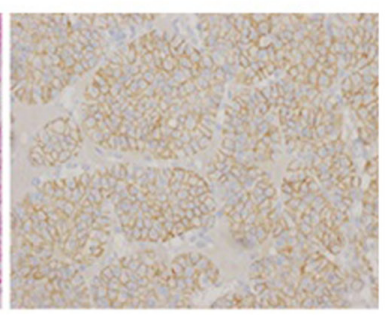

$\mathrm{H}$

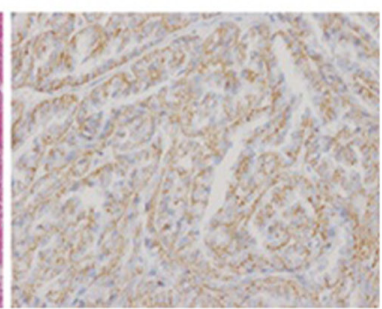

Figure 1. Eosinophilic kidney tumors and TRPM4 staining patterns (A) H\&E staining (magnification x100) and (B) negative immunoexpression of TRPM4 (magnification x100) in clear cell renal cell carcinoma. (C) H\&E staining (magnification x100) and (D) diffuse membranous granular immunoexpression of TRPM4 (magnification, x200) in renal oncocytoma. (E) H\&E staining (magnification x100) and (F) focal membranous expression of TRPM4 (magnification x 100) in chromophobe renal cell carcinoma. (G) H\&E staining (magnification x40) and (H) basolateral granular expression of TRPM4 (magnification x200) in papillary renal cell carcinoma type 2. TRPM4, transient receptor potential cation channel subfamily M member 4.

A

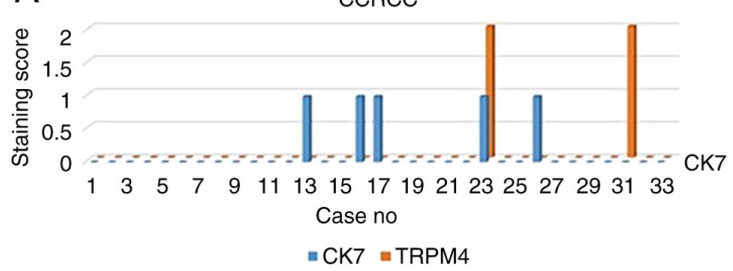

C

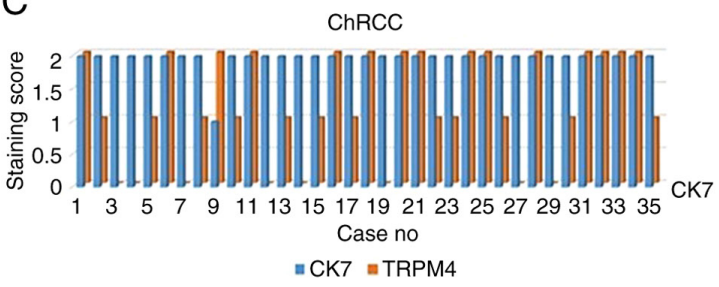

B
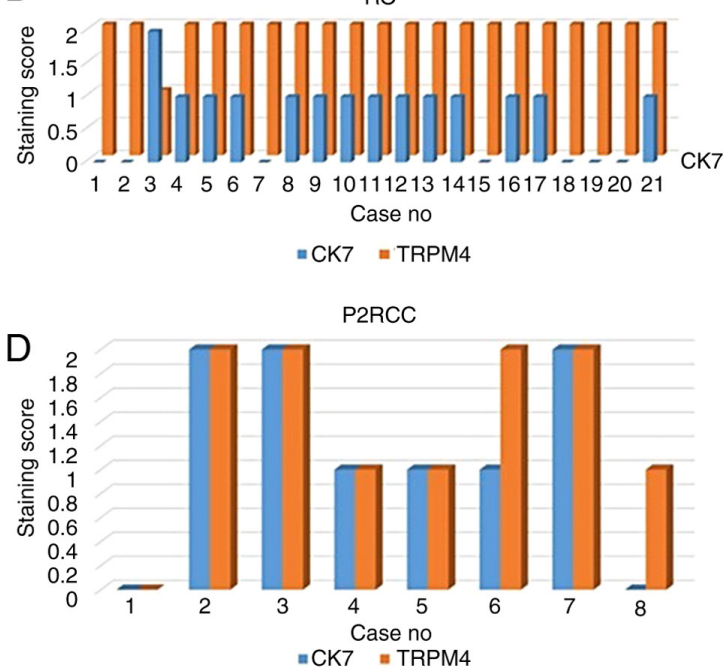

Figure 2. Comparison of TRPM4 and CK7 staining in patients with CCRCC, RO, ChRCC and P2RCC. (A) Comparison of TRPM4 and CK7 staining in patients with CCRCC. (B) Comparison of TRPM4 and CK7 staining in patients with RO. (C) Comparison of TRPM4 and CK7 staining in patients with ChRCC. (D) Comparison of TRPM4 and CK7 staining in patients with P2RCC. Y-axis, Prevalence of staining; 0, negative; 1, focal positive; and 2, diffuse positive. X-axis: Number of cases stained with CK7 and TRPM4 immunohistochemical staining TRPM4, transient receptor potential cation channel subfamily M member 4; CK7, cytokeratin 7; CCRCC, clear cell renal cell carcinoma; RO, renal oncocytoma; ChRCC, chromophobe renal cell carcinoma; P2RCC, papillary renal cell carcinoma type 2 .

its reliability can be increased to a more significant level with other markers and large series.

\section{Acknowledgements}

Not applicable.

\section{Funding}

No funding was received.

\section{Availability of data and materials}

The datasets used and/or analyzed during the current study are available from the corresponding author on reasonable request.

\section{Authors' contributions}

GÇ, PY and NŞ conceived and designed the study. GÇ, NŞ, and $\mathrm{BD}$ acquired the data. GÇ and $\mathrm{ZG}$ analyzed and interpreted the data. GÇ, NŞ and ZG confirm the authenticity of the 
raw data. GÇ and NŞ wrote the manuscript. All authors read and approved the final manuscript.

\section{Ethics approval and consent to participate}

The present study was approved by the Ethical Committee of Bezmialem Vakif University Hospital (Istanbul, Turkey; approval no. 11-202), and written informed consent was obtained from each patient.

\section{Patient consent for publication}

Not applicable.

\section{Competing interests}

The authors declare that they have no competing interests.

\section{References}

1. Iczkowski KA and Czaja RC: Eosinophilic kidney tumors: Old and new. Arch Pathol Lab Med 143: 1455-1463, 2019.

2. Kryvenko ON, Jorda M, Argani P and Epstein JI: Diagnostic approach to eosinophilic renal neoplasms. Arch Pathol Lab Med 138: 1531-1541, 2014.

3. Guinamard R, Sallé L and Simard C: The non-selective monovalent cationic channels TRPM4 and TRPM5. Adv Exp Med Biol 704: 147-171, 2011.

4. Vennekens R and Nilius B: Insights into TRPM4 function, regulation and physiological role. Handb Exp Pharmacol 179: 269-285, 2007

5. Prevarskaya N, Skryma R, Bidaux G, Flourakis M and Shuba Y: Ion channels in death and differentiation of prostate cancer cells. Cell Death Differ 14: 1295-1304, 2007.

6. Kappel S, Stokłosa P, Hauert B, Ross-Kaschitza D, Borgström A, Baur R, Galván JA, Zlobec I and Peinelt C: TRPM4 is highly expressed in human colorectal tumor buds and contributes to proliferation, cell cycle, and invasion of colorectal cancer cells. Mol Oncol 13: 2393-2405, 2019.

7. Holzmann C, Kappel S, Kilch T, Jochum MM, Urban SK, Jung V, Stöckle M, Rother K, Greiner M and Peinelt C: Transient receptor potential melastatin 4 channel contributes to migration of androgen-insensitive prostate cancer cells. Oncotarget 6 : 41783-41793, 2015.

8. Gao Y and Liao P: TRPM4 channel and cancer. Cancer Lett 454: 66-69, 2019.

9. Shapovalov G, Ritaine A, Skryma R and Prevarskaya N: Role of TRP ion channels in cancer and tumorigenesis. Semin Immunopathol 38: 357-369, 2016.

10. Monteith GR, McAndrew D, Faddy HM and Roberts-Thomson SJ: Calcium and cancer: Targeting $\mathrm{Ca}^{2+}$ transport. Nat Rev Cancer 7 : 519-530, 2007.

11. Schattling B, Steinbach K, Thies E, Kruse M, Menigoz A, Ufer F, Flockerzi V, Brück W, Pongs O, Vennekens R, et al: TRPM4 cation channel mediates axonal and neuronal degeneration in experimental autoimmune encephalomyelitis and multiple sclerosis. Nat Med 18: 1805-1811, 2012.

12. Tosun C, Kurland DB, Mehta R, Castellani RJ, deJong JL, Kwon MS, Woo SK, Gerzanich V and Simard JM: Inhibition of the Sur1-Trpm4 channel reduces neuroinflammation and cognitive impairment in subarachnoid hemorrhage. Stroke 44: 3522-3528, 2013.
13. Mehta RI, Tosun C, Ivanova S, Tsymbalyuk N, Famakin BM, Kwon MS, Castellan RJ, Gerzanich V and Simard JM: Surl-Trpm4 cation channel expression in human cerebral infarcts. J Neuropathol Exp Neurol 74: 835-849, 2015.

14. Qin F, Lao L, Huang M, Tan H, Jin X, Ma X and Zeng J: Evaluation of the TRPM protein family as potential biomarkers for various types of human cancer using public database analyses. Exp Ther Med 20: 770-785, 2020.

15. Holzmann C, Kappel S, Kilch T, Jochum MM, Urban SK, Jung V, Stöckle M, Rother K, Griner M and Peinelt C: Transient receptor potential melastatin 4 channel contributes to migration of androgen-insensitive prostate cancer cells. Oncotarget 8: 41783-41793, 2015

16. Ceylan GG, Önalan EE, Kuloğlu T, Aydoğ G, Keleş İ, Tonyal Ş and Ceylan C: Potential role of melastatin-related transient receptor potential cation channel subfamily $\mathrm{M}$ gene expression in the pathogenesis of urinary bladder cancer. Oncol Lett 12: 5235-5239, 2016

17. Sozucan Y, Kalender ME, Sari I, Suner A, Oztuzcu S, Arman K, Yumrutas O, Bozgeyik I, Cengiz B, Igci YZ, et al: TRP genes family expression in colorectal cancer. Exp Oncol 37: 208-212, 2015.

18. Narayan G, Bourdon V, Chaganti S, Arias-Pulido H, Nandula SV, Rao PH, Gissmann L, Dürst M, Schneider A, Pothuri B, et al: Gene dosage alterations revealed by cDNA microarray analysis in cervical cancer: Identification of candidate amplified and overexpressed genes. Genes Chromosomes Cancer 40: 373-384, 2007.

19. Loo SK, Ch'ng ES, Md Salleh MS, Banham AH, Pedersen LM, Møller MB, Green TM and Wong KK: TRPM4 expression is associated with activated B cell subtype and poor survival in diffuse large B cell lymphoma. Histopathology 71: 98-111, 2017.

20. Chen J, Luan Y, Yu R, Zhang Z, Zhang J and Wang W: Transient receptor potential (TRP) channels, promising potential diagnostic and therapeutic tools for cancer. Biosci Trends 8: 1-10, 2014.

21. Ashida S, Nakagawa H, Katagiri T, Furihata M, Liizumi M, Anazawa Y, Tsunoda T, Takata R, Kasahara K, Miki T, et al: Molecular features of the transition from prostatic intraepithelial neoplasia (PIN) to prostate cancer: Genome-wide gene-expression profiles of prostate cancers and PINs. Cancer Res 64: 5963-5972, 2004.

22. Berg KD, Soldini D, Jung M, Dietrich D, Stephan C, Jung K, Dietel M, Vainer B and Kristiansen G: TRPM4 protein expression in prostate cancer: A novel tissue biomarker associated with risk of biochemical recurrence following radical prostatectomy. Virchows Arch 468: 345-355, 2016.

23. Trpkov $\mathrm{K}$ and Hes O: New and emerging renal entities: $A$ perspective post-WHO 2016 classification. Histopathology 74: 31-59, 2019.

24. Liu L, Qian J, Singh H, Meiers I, Zhou X and Bostwick DG: Immunohistochemical analysis of chromophobe renal cell carcinoma, renal oncocytoma, and clear cell carcinoma: An optimal and practical panel for differential diagnosis. Arch Pathol Lab Med 131: 1290-1297, 2007.

25. Delahunt B and Eble JN: Papillary renal cell carcinoma: A clinicopathologic and mmunohistochemical study of 105 tumors. Mod Pathol 10: 537-544, 1997.

26. Hong X and Yu JJ: MicroRNA-150 suppresses epithelial mesenchymal transition, invasion and metastasis in prostate cancer through the TRPM4-mediated $\beta$-catenin signaling pathway. Am J Physiol Cell Physiol 316: C463-C480, 2019.

27. Wong KK and Hussain FA: TRPM4 is overexpressed in breast cancer associated with estrogen response and epithelialmesenchymal transition gene sets. PLoS One 15: e0233884, 2020. 